\title{
Shenzhen stroke emergency map improves access to rt-PA for patients with acute ischaemic stroke
}

\author{
Shisheng Ye, ${ }^{01}$ Shiyu Hu, ${ }^{1}$ Zhihao Lei, ${ }^{1}$ Zhichao Li, ${ }^{1}$ Weiping Li, ${ }^{1}$ Yi Sui, ${ }^{2}$ Lijie Ren ${ }^{1}$
}

To cite: Ye S, Hu S, Lei Z, et al. Shenzhen stroke emergency map improves access to rtPA for patients with acute ischaemic stroke. Stroke and Vascular Neurology 2019;4: e000212. doi:10.1136/svn2018-000212

- Additional material is published online only. To view please visit the journal online (http://dx.doi.org/10.1136/svn2018-000212).

SY and SH contributed equally.

Received 14 November 2018 Revised 12 February 2019 Accepted 13 February 2019 Published Online First 21 June 2019
Check for updates

(C) Author(s) (or their employer(s)) 2019. Re-use permitted under CC BY-NC. No commercial re-use. See rights and permissions. Published by BMJ.

${ }^{1}$ Department of Neurology, Shenzhen University First Affiliated Hospital, Shenzhen Second People's Hospital, Shenzhen, China ${ }^{2}$ Department of Neurology, Shenyang First People's Hospital, Shenyang Medical College, Shenyang, China

Correspondence to Professor Lijie Ren; 13631605966@126.com

\section{ABSTRACT}

Prehospital delay is one of the major causes of low rate of intravenous recombinant tissue plasminogen activator (rt-PA) thrombolysis for acute ischaemic stroke in China. Regional emergency systems have been proven a successful approach to improve access to thrombolysis. Shenzhen is a high population density city with great geographical disparity of healthcare resources, leading to limited access to rt-PA thrombolysis for most patients with acute ischaemic stroke. To improve rapid access to rt-PA thrombolysis in Shenzhen, a Shenzhen stroke emergency map was implemented by Shenzhen healthcare administrations. This map comprised certification of qualified local hospitals, identification of patients with stroke, acute stroke transport protocol and maintenance of the map. We conducted a retrospective observational study to compare consecutive patients with acute stroke arriving at qualified local hospitals before and after implementation of the Shenzhen stroke emergency map. After implementation of the map, the rate of patients receiving rt-PA thrombolysis increased from $8.3 \%$ to $9.7 \%(\mathrm{p}=0.003)$, and the rate of patients treated with endovascular thrombectomy increased from $0.9 \%$ to $1.6 \%(p<0.001)$. Sixteen of 20 hospitals have an increase in the number of patients with stroke treated with rt-PA thrombolysis. The median time between receipt of the call and arrival on the scene reduced significantly $(17.0 \mathrm{~min}$ vs 9.0 min, $p<0.001)$. In Shenzhen Second People's Hospital, the median onset-to-needle time and door-to-needle time were reduced ( $175.5 \mathrm{~min}$ vs $149.5 \mathrm{~min}, \mathrm{p}=0.039$; $71.5 \mathrm{~min}$ vs $51.5 \mathrm{~min}, \mathrm{p}<0.001)$. No statistically significant differences were found in the proportion of rt-PA-treated patients within various geographical distances. Currently, there are more than 40 cities in China implementing a stroke emergency map. The Shenzhen stroke emergency map improves access to rt-PA thrombolysis for acute ischaemic stroke, and the novel model has been expanded to multiple areas in China. Future efforts should be conducted to optimise the stroke emergency map.

\section{INTRODUCTION}

Stroke is the third leading cause of death and adult disability in most Western countries, ${ }^{1}$ but is the leading in China. ${ }^{2}$ In China, stroke care costs approximately $¥ 40$ billion per year, 10 times higher than cardiovascular diseases, ${ }^{3}$ which has a significant impact on national healthcare expenditures and economy. Acute ischaemic stroke (AIS) accounts for approximately $70 \%$ of all types of stroke, and the most effective treatment approach is early reperfusion therapy. Currently, intravenous recombinant tissue plasminogen activator (rt-PA) is strongly recommended for selected AIS in early reperfusion therapy within 4.5 hours of symptom onset, ${ }^{4}$ with earlier administration associated with bigger proportional benefit. ${ }^{5}$ However, the rate of intravenous rt-PA use remains very low $(1.6 \%)$ in China. ${ }^{6}$ One of the major causes is the significant prehospital delays, making a multitude of patients with AIS ineligible for reperfusion therapies. In a study that involved 62 hospitals in 37 cities across China, the median time of prehospital delay was 15.0 hours. $^{7}$ Based on these considerations, different approaches have been explored to reduce prehospital delay to improve access to thrombolysis. Regional emergency systems have been successful in the rapid access to thrombolysis in London, Los Angeles and other metropolises in the past decades. ${ }^{8-11}$ Subsequently, guidelines emphasise the necessity of developing regional systems of stroke care to improve access to thrombolysis. ${ }^{12} 13$

Shenzhen is the fourth largest city in China and has a population of approximately 20 million residing in $700 \mathrm{~km}^{2}$. On the basis of the Monitoring System of New Stroke Cases in Shenzhen data, 16739 new stroke cases occurred in 2013, increasing at a rate of $10 \%$ a year. Most healthcare resources are allocated in south-western Shenzhen, resulting in geographical disparity due to unbalanced allocation of healthcare system resources. Therefore, access to rt-PA therapy for a majority of patients with AIS in Shenzhen is still limited. To address this issue, a stroke emergency map was established in November 2016, as part of regional stroke systems of care. A stroke emergency map is a regional emergency system, not a simple geographical map, comprising certification of qualified local hospitals, identification of patients with stroke, acute stroke transport protocol and maintenance of the map. The aim of this map was to provide 
rapid recognition and transport of appropriate patients with AIS directly to appropriate hospitals (sometimes bypassing the nearest hospitals) that can provide intravenous thrombolysis and endovascular thrombectomy. The objective of this study was to assess the effectiveness of the Shenzhen stroke emergency map to optimise access to rt-PA for patients with AIS.

\section{METHODS}

Study design

This is a retrospective observational study using data from 1 January 2016 to 31 December 2017, comparing the thrombolysis rate and thrombectomy rate as well as emergency medical services (EMS) system response time before (1 January 2016-31 December 2016) and after (1 January 2017-31 December 2017) implementation of the Shenzhen stroke emergency map.

\section{Certification of qualified local hospitals}

Ordered by healthcare administrations, the Stroke Care Quality Management Center was established, as an independent group, providing formal qualified local hospital certification. Although stroke centre designations exist (such as acute stroke-ready hospital, primary stroke centre (PSC) and comprehensive stroke centre (CSC)), the committee of experts modified the designation to meet the circumstances in Shenzhen based on recommendations for PSC and CSC..$^{14}$ Certification criteria for qualified local hospitals of the Shenzhen stroke emergency map were organised around six major aspects of stroke care, including qualification of medical institutions, department settings, infrastructure, staffing, programmes to diagnose and treat patients with stroke, as well as management system (online supplementary file 1). After the written assessment material reviews, onsite certification reviews were conducted by experts with expertise in stroke care. To achieve qualified local hospital certification, hospitals had to score more than 80 points. Qualified local hospitals that successfully met the certification criteria were awarded certification for a 3 -year period. To maintain certification, the onsite review will be conducted every year. There are 74 1-2-0 network hospitals in Shenzhen, but only 23 hospitals have acute stroke care capability to apply for certification. Finally, 20 hospitals in 8 districts achieved qualified local hospital certification.

\section{Identification of patients with stroke}

The first major issue for EMS staff ('120' staff) is identifying the patients who should be transported to a qualified local hospital under the acute stroke triage protocol. After the identification of patients with stroke, EMS staff should initiate appropriate treatment on the scene, determine the most appropriate hospital, notify the receiving hospital and transport patients rapidly. With the support of Shenzhen healthcare administrations, the EMS training was developed by collaboration of EMS directors, stroke centre directors, and experts from
EMS and stroke centres. Training was provided to EMS communicators/dispatchersand staff about the knowledge of stroke by neurologists and educational materials which reflect current published guidelines for prehospital management of patients with AIS. The training ensures the EMS communicators can recognise the signs and symptoms of stroke as reported by callers, and prioritise ambulance dispatch for patients with acute stroke. More than 50 EMS staff were given training to identify potential patients with stroke on the scene, and perform the shortened National Institutes of Health Stroke Scale (NIHSS) or Cincinnati Prehospital Stroke Scale (CPSS) assessment rapidly, assisting them in rendering a primary diagnosis of stroke. To ensure optimal EMS for patients with stroke, these stroke training efforts were planned to be conducted periodically (once every month in the first half year, once every 3 months in the second half year and per 6 months later). Also, stroke centre leaders and EMS directors would adjust the frequency of stroke training based on continuous quality improvement projects.

\section{Acute stroke triage protocol}

An acute stroke transport protocol within a region must consider several factors, such as time from onset (or last known well), transport distances, transport time, the capability of hospitals and patient preference. Shenzhen 1-2-0 First Aid Network consists of 74 network hospitals, 81 first aid stations and 142 network ambulances. The 1-2-0 first-aid call centre is the only system that responds to all 120 calls in the city, providing central coordination of all ambulances service 24/7 (24 hours per day, 7 days per week) and ensuring rapid and efficient dispatch and transfer. All dispatch and transfers follow the principle of proximity, dispatching the nearest available ambulance and transferring to the nearest qualified local hospital with stroke care capability certification. Furthermore, the process of hospital decision-making must consider the right of patient and patient family members to discuss with the EMS staff which hospital they want to be transported to, avoiding unethical or financially driven behaviours. The flow diagram can be seen in figure 1 .

\section{Maintenance of the map}

After the stroke emergency map was established, a programme for stroke care quality improvement was established. Such a programme will review the following data: number of disease-specific patients, method of treatment, timeline for providing treatment and assessment of outcome, implemented by the Stroke Care Quality Management Center. All the qualified local hospitals must collect the data on the performance of quality measurements and submit them quarterly to the Stroke Care Quality Management Center. The programme is financed by Shenzhen healthcare administrations.

\section{Statistical analysis}

Continuous variables were presented as mean $\pm \mathrm{SD}$ or median (IQR), and categorical variables were presented 
Acute stroke triage protocol

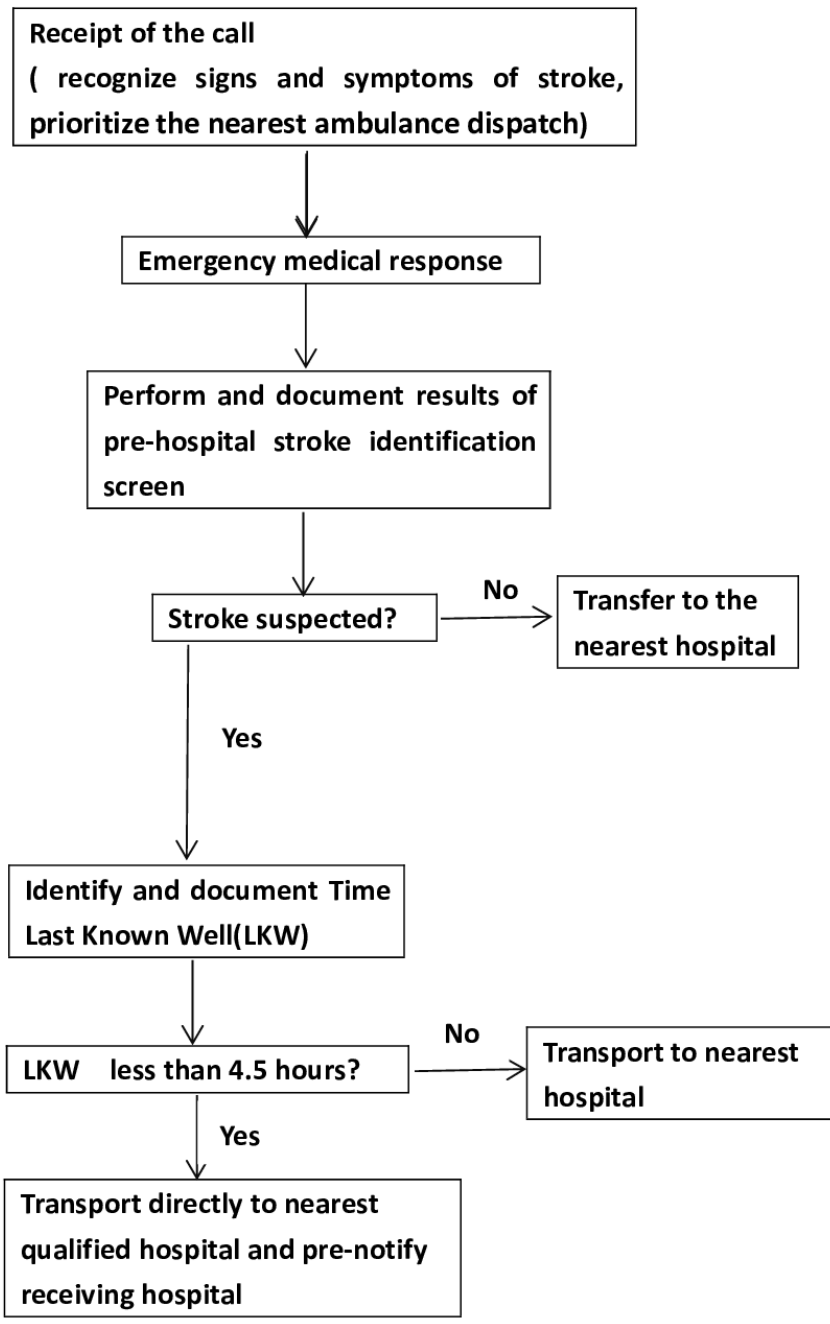

Figure 1 Flow of acute stroke triage protocol. The emergency medical service (EMS) communicators receive the call, recognise the signs and symptoms of stroke, as well as prioritise the nearest ambulance dispatch. After the ambulance arrival on the scene, EMS staff perform and document the results of prehospital stroke identification screen. If stroke is suspected, the time last known well (LKW) is identified and documented. If the LKW is less than 4.5 hours, the patient is transported directly to the nearest qualified hospital and the receiving hospital prenotified. If not, the patient will be transported to the nearest hospital.

as frequencies. Comparisons were performed on thrombolysis rate, thrombectomy rate, median time between receipt of the call and arrival on the scene, median onset-to-needle time (ONT), median door-to-needle time (DNT), and the rate of rt-PA-treated patients within various distances before and after map implementation using independent Student's t-test, Mann-Whitney U test and Pearson's $\mathrm{X}^{2}$ test as appropriate. All of the statistical analyses were performed using SPSS V.22.0. A $p$ value $<0.05$ was considered statistically significant (two-sided).
RESULTS

The stroke emergency map was released in Shenzhen in November 2016 (figure 2), which only showed hospitals that have intravenous thrombolysis capability. The map showed some large geographical areas with high population density that have no one qualified hospital, such as the northern part of Baoan District, Pingshan District and Dapeng District. Figure 3 is the updated version of the map released in 2018. It clearly showed the hospitals that have thrombolysis and thrombectomy capabilities, newly qualified hospitals (Shenzhen Baoan Second People's Hospital and Shenzhen Baoan Traditional Chinese Medicine Hospital), and new sites undergoing construction or planned (Shenzhen Songgang People's Hospital, Shenzhen Pingshan New District People's Hospital and Shenzhen Dapeng Kuichong People's Hospital).

The stroke emergency map initiative resulted in an immediate increase in the absolute number of patients with acute stroke treated with rt-PA thrombolysis from 568 to 802 , corresponding to an increase in the rate of rt-PA thrombolysis from $8.3 \%$ to $9.7 \%$ ( $p=0.003$ ) (table 1 ). The number of patients treated with endovascular thrombectomy increased from 60 to 136, corresponding to an increase in the rate of endovascular thrombectomy from $0.9 \%$ to $1.6 \% \quad(\mathrm{p}<0.001)$ (table 1$)$. Furthermore, there was a significant reduction in the median time between receipt of the call and arrival on the scene (17.1 min vs $9.7 \mathrm{~min}, \mathrm{p}<0.001$ ) (table 1). In Shenzhen Second People's Hospital, the median ONT and DNT for rt-PA-treated patients decreased by $26 \mathrm{~min}$ and $20 \mathrm{~min}$, respectively ( $175.5 \mathrm{~min}$ vs $149.5 \mathrm{~min}, \mathrm{p}=0.039 ; 71.5 \mathrm{~min}$ vs $51.5 \mathrm{~min}, \mathrm{p}<0.001$ ) (table 1 ). However, the proportion of the rt-PA-treated patients within various geographical distances of Shenzhen Second People's Hospital did not differ significantly before and after the implementation of the map (table 1). Sixteen of 20 hospitals had an increase in the number of rt-PA-treated patients, especially Shenzhen Longgang People's Hospital, University of Chinese Academy of Sciences Shenzhen Hospital and Peking University Shenzhen Hospital, while 4 of 20 hospitals had the opposite result (figure 4). Moreover, the hospitals that had an increase in the number of rt-PA-treated patients had no significant difference in stratification by hospital designation (thrombolysis-capable only vs thrombolysis-capable and thrombectomy-capable) (7/9 vs $9 / 11$, $\mathrm{p}=0.822)$. Such stroke emergency maps have been established in multiple areas in China. Currently, there are more than 40 cities in China establishing an stroke emergency map.

\section{DISCUSSION}

This stroke emergency map was immediately successful in improving rt-PA thrombolysis access for eligible patients with AIS, increasing rt-PA thrombolysis rates in Shenzhen and demonstrating the need for integrated systems of stroke care. Most hospitals had an increase 


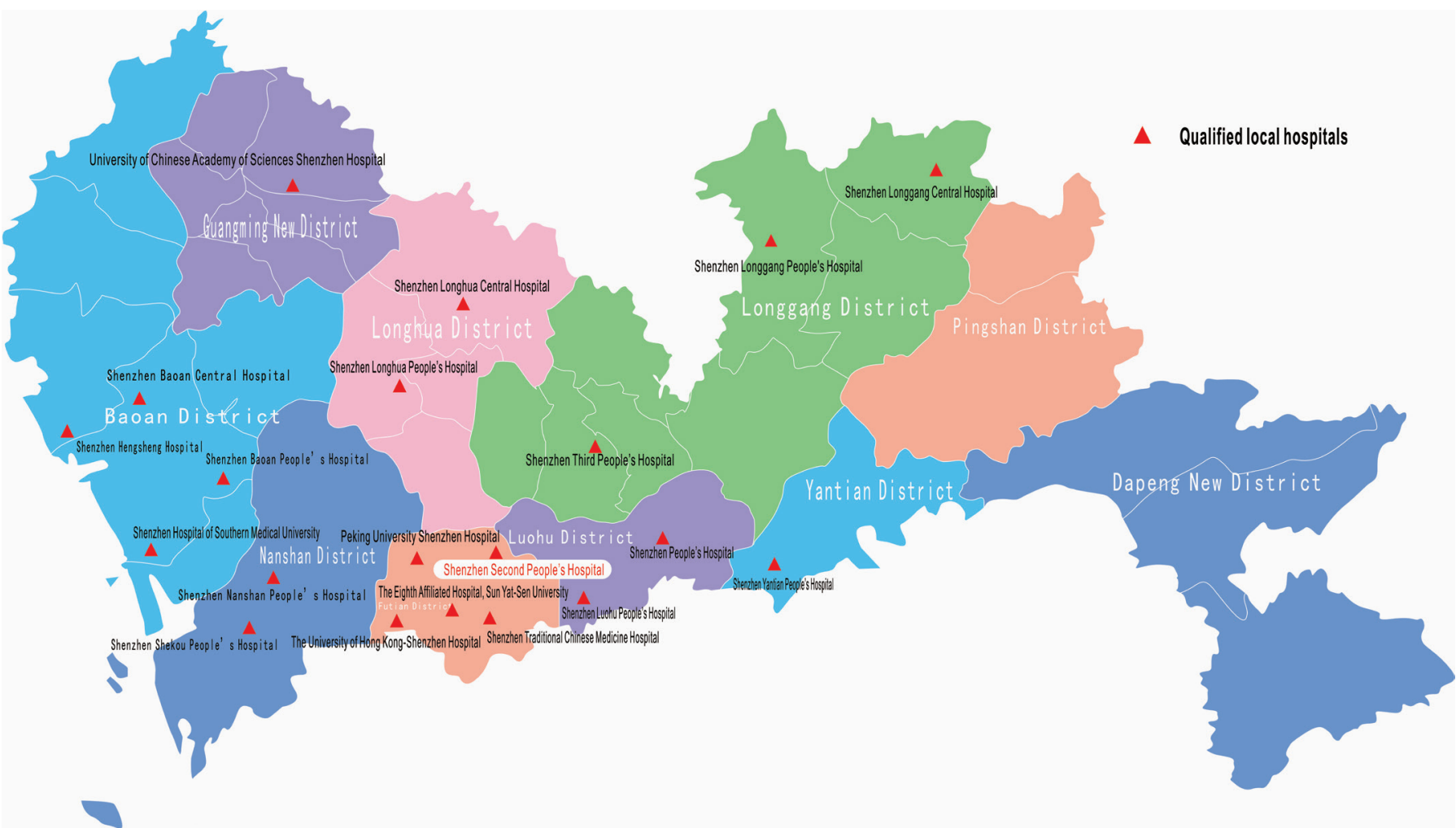

Figure 2 Initial version of the stroke emergency map. The map only showed hospitals that have thrombolysis capabilities. It showed some large geographical areas with high population density that have no one qualified hospital, such as the northern part of Baoan District, Pingshan District and Dapeng District. Red ' $\mathbf{\Delta}$ ' indicated qualified hospitals that have stroke care capabilities.

in the number of patients treated with rt-PA, while four hospitals even had an opposite result, probably reflecting the transport of more patients to nearer qualified hospitals. Therefore, EMS must determine the hospital transport destinations based on the triage protocol, avoiding competition among hospitals for patients. As is shown in figure 2, Shenzhen Longgang People's Hospital and the University of Chinese Academy of Sciences Shenzhen Hospital serve in a large geographical area where there is only one qualified hospital receiving the majority of patients in this area, leading to a great increase in the number of rt-PA-treated patients. The elapsed time from receipt of the call to arrival on the scene (overall EMSsystem response time) was shorter after implementation of stroke emergency map, implying more rapid identification of patients with stroke and ambulance dispatch. For patients receiving rt-PA in Shenzhen Second People's Hospital, both prehospital and in-hospital delay (ONT and DNT) decreased, probably resulting from improvement of overall EMSsystem response and in-hospital workflow due to prehospital hospital notification. However, the proportion of patients transferred from long distance did not decrease after implementation of the map, probably because Shenzhen Second People's Hospital with high-level stroke care capability attracted long-distance patients to require transportation to farther hospitals. Therefore, EMS staff training should include prehospital patient education about the importance of therapeutic time window. Overall, ongoing quality improvement efforts should be made to ensure desired results.

The successful implementation of an stroke emergency map in Shenzhen was generalised to other cities with a positive outcome to increase accessibility to rt-PA. The growing stroke emergency map in China meets the great need of a large population and is extremely important for both individuals and society in reducing stroke morbidity and mortality. It could have other functions, such as guiding local policy makers to establish new qualified stroke centres in regional areas that lack stroke care resources (such as Shenzhen Songgang People's Hospital, Shenzhen Pingshan New District People's Hospital and Shenzhen Dapeng Kuichong People's Hospital), promoting establishment and improvement of prehospital emergency network, promoting the formation of stroke prehospital triage system and improving awareness of patients of nearby hospitals with stroke care capabilities. With the increase of the map across China, establishing an organisation for developing entry criteria to provide official certification process and unified management of all maps is the critical next step.

Recent evidence on the benefit of endovascular thrombectomy in patients presenting within 16-24hours from time last known well has promoted to increase access to hospitals that provide efficacious therapy rapidly and safely. ${ }^{16} 17$ A randomised clinical trial showed that interhospital transfer is the most important cause of 


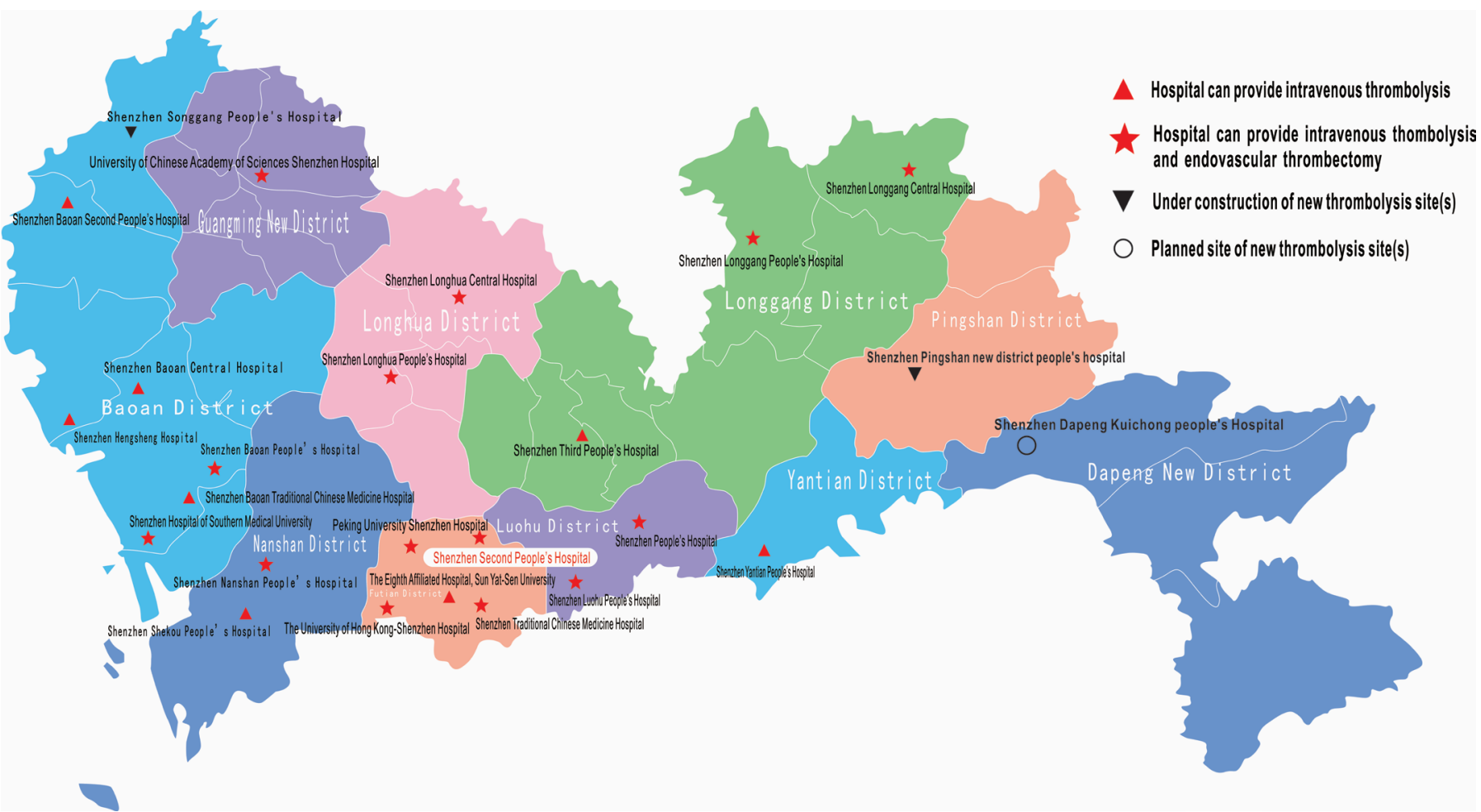

Figure 3 Updated version of the stroke emergency map. The map showed hospitals that have thrombolysis and thrombectomy capabilities, newly qualified hospitals (Shenzhen Baoan Second People's Hospital and Shenzhen Baoan Traditional Chinese Medicine Hospital), and new sites undergoing construction or planned (Shenzhen Songgang People's Hospital, Shenzhen Pingshan New District People's Hospital and Shenzhen Dapeng Kuichong People's Hospital). Red ' $\boldsymbol{\Delta}$ ' indicated hospitals that have thrombolysis capabilities. Red ' $\star$ ' indicated hospitals that have both thrombolysis and thrombectomy capabilities. Black ' $\boldsymbol{\nabla}$ ' indicated a new site under construction. ' $O$ ' indicated a new planned site.

endovascular treatment delays (the Multicenter Randomized Clinical Trial of Endovascular Treatment for Acute Ischemic Stroke in the Netherlands).$^{18}$ Furthermore, the interhospital transfer delay is mainly attributable to doorin-door-out (DIDO) times at the PSC. ${ }^{19}$ The benefit of endovascular thrombectomy for AIS is also significantly

Table 1 Comparison of patients with acute ischaemic stroke before and after implementation of the stroke emergency map

\begin{tabular}{|c|c|c|c|}
\hline & Before map & After map & $P$ value \\
\hline $\begin{array}{l}\text { Number }(\%) \text { of patients treated with rt-PA } \\
\text { thrombolysis }\end{array}$ & $568 / 6843(8.3)$ & 802/8268 (9.7) & 0.003 \\
\hline $\begin{array}{l}\text { Number (\%) of patients treated with endovascular } \\
\text { thrombectomy }\end{array}$ & $60 / 6843(0.9)$ & $136 / 8268(1.6)$ & $<0.001$ \\
\hline $\begin{array}{l}\text { Median time between receipt of the call and } \\
\text { arrival on the scene (min) (IQR ) }\end{array}$ & $17.0(7.0)$ & $9.0(3.8)$ & $<0.001$ \\
\hline Median onset-to-needle time (min) (IQR ) & $175.5(67.8)$ & $149.5(71.8)$ & 0.039 \\
\hline Median door-to-needle time (min) (IQR ) & $71.5(43.8)$ & $51.5(26.8)$ & $<0.001$ \\
\hline \multicolumn{4}{|c|}{ Number (\%) of rt-PA-treated patients within various distances of Shenzhen Second People's Hospital } \\
\hline$\leq 3 \mathrm{~km}$ & $10 / 56(17.9)$ & 9/58 (15.5) & 0.738 \\
\hline $3-5 \mathrm{~km}$ & 11/56 (19.6) & $10 / 58(17.2)$ & 0.741 \\
\hline $5-10 \mathrm{~km}$ & 15/56 (26.8) & $15 / 58(25.9)$ & 0.911 \\
\hline$>10 \mathrm{~km}$ & 20/56 (35.7) & $24 / 58(41.4)$ & 0.535 \\
\hline
\end{tabular}

In 20 qualified hospitals, the number of patients with acute stroke treated with rt-PA thrombolysis increased from 568 to 802 , and the rate of rt-PA thrombolysis increased from $8.3 \%$ to $9.7 \%$. The number of patients treated with endovascular thrombectomy increased from 60 to 136 , and the rate of patients treated with endovascular thrombectomy increased from $0.9 \%$ to $1.6 \%$. Furthermore, the median time between receipt of the call and arrival on the scene decreased significantly. In Shenzhen Second People's Hospital, the median onset-to-needle time and door-to-needle time for rt-PA-treated patients decreased by $26 \mathrm{~min}$ and $20 \mathrm{~min}$, respectively. The proportion of rt-PA-treated patients within various geographical distances of Shenzhen Second People's Hospital did not differ significantly. rt-PA, recombinant tissue plasminogen activator. 


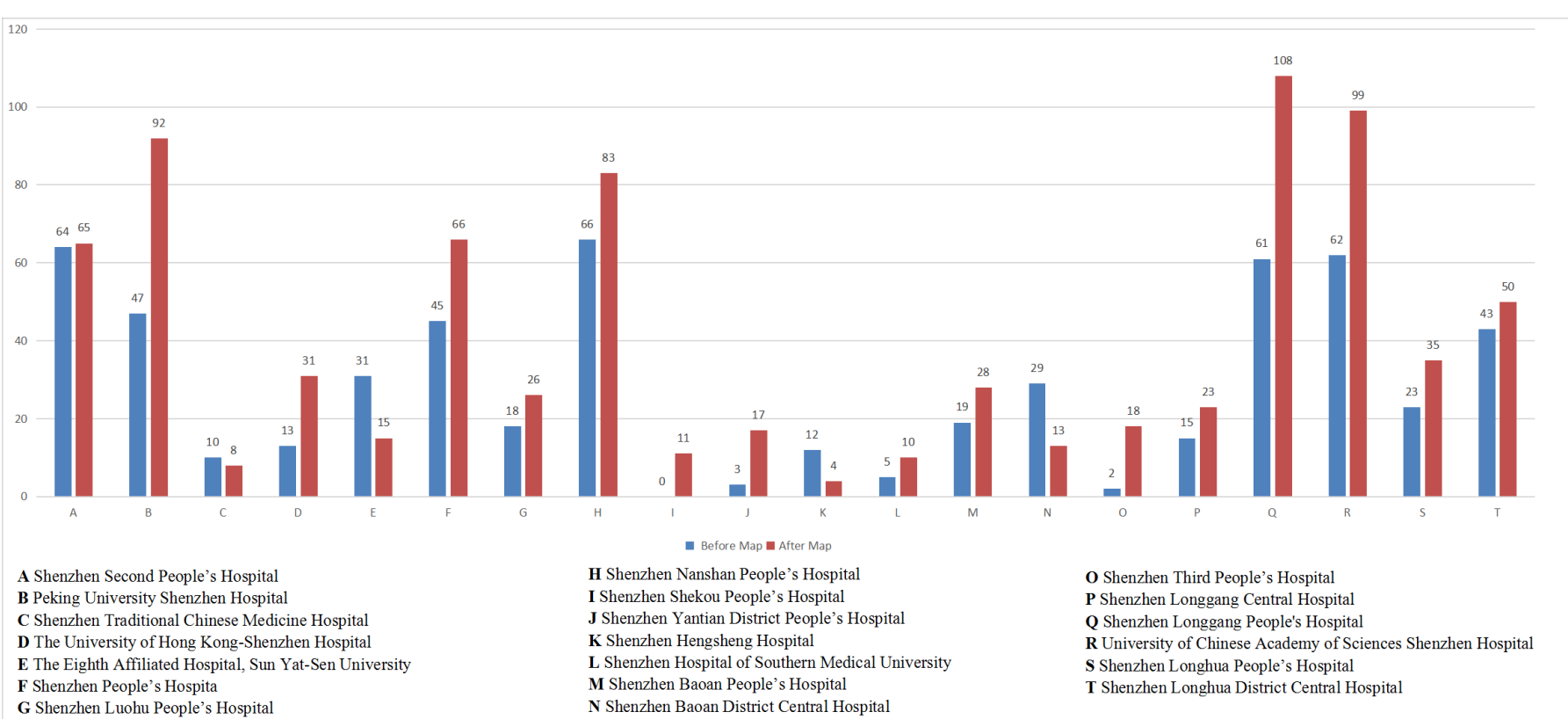

Figure 4 Comparison of the number of patients treated with rt-PA thrombolysis in 20 qualified hospitals. Sixteen of 20 hospitals had an increase in the number of rt-PA-treated patients, especially Shenzhen Longgang People's Hospital, University of Chinese Academy of Sciences Shenzhen Hospital and Peking University Shenzhen Hospital, while 4 of 20 hospitals had the opposite result. rt-PA, recombinant tissue plasminogen activator.

time-dependent, ${ }^{20}$ and making every effort to avoid intertransfers and reduce intertransfer delay is a direction for future exploration. For patients with suspected large vessel occlusion (LVO), EMS must determine whether to select bypassing a closer thrombolysis-capable hospitals directly to a thrombectomy-capable hospital (mothership model), or transferring to thrombectomy-capable hospital after initiating intravenous thrombolysis at a closer thrombolysis-capable hospitals (drip-and-ship model). The Lifeline Stroke Committee craft a consensus algorithm that recommends transport to the closest PSC or acute stroke-ready hospital if the additional transport delay to CSC is more than $15 \mathrm{~min}^{21}$ However, the DIDO times are generally prolonged in China due to uneven level of stroke centres (or acute stroke-ready hospital), lack of corresponding interhospital transfer workflow, incentives and continuous quality improvement projects. Therefore, bypassing a nearby thrombolysis-capable centre directly to a thrombectomy-capable hospital may be reasonable in some circumstances. In the new 2018 version of the map, hospitals that have thrombectomy capabilities are clearly indicated. Hence, after identifying prehospital patients with suspected LVO using stroke severity scales, EMS can directly transfer patients to thrombectomy-capable hospitals for endovascular recanalisation therapy. Efforts are now under way in EMS training on performing stroke severity scales to assess potential LVO.

Increasingly, telemedicine has been proven useful in prehospital stroke evaluation and triage. A retrospective observational study compared clinical outcomes of acute endovascular treatment between patients with AIS transferred after telemedicine consultation and those primarily admitted to tertiary hospitals. The patients treated by telestroke and those primarily admitted observed similar rates of endovascular reperfusion and beneficial functional outcomes. ${ }^{22}$ Furthermore, in Germany, the uses of telemedicine for stroke involve a hub-and-spoke telestroke network, in which the hub hospital provides telestroke service $24 / 7$ to spoke hospitals without neurological or radiological services. ${ }^{23}$ Within the stroke emergency map, telemedicine may play a role in identification of appropriate patients who are in need of emergent transfer to thrombectomy-capable hospitals, while those with suspected LVO are primarily admitted to thrombolysis-capable hospitals.

Early recognition of stroke warning signs is essential for seeking emergency care immediately. Unfortunately, data from three cities in China showed that public awareness of stroke symptoms and treatment remains poor, and only $18.8 \%$ used the EMS. ${ }^{24}$ Therefore, it is critical to improve public awareness of stroke and stroke recognition to trigger EMS in a rapid manner. Public stroke education is aimed at patients with stroke and their family members, especially younger generation, and requires repetition for a continuing impact. Stroke 120 as a novel stroke educational tool can be easily remembered even by those with no high education. ${ }^{25}$ One public stroke education programme combining stroke emergency map and Stroke 120 is now running to improve stroke awareness and EMS utilisation in Shenzhen.

There were several limitations to our study. We identified the beginning of implementation period as the year 2017, not the actual dates of releasing the map, which had a 1-month trial adjustment period. Also, as 
a retrospective study, we lacked completeness of databases, because the data were not primarily collected for study purposes. First, although EMS staff were provided training to identify potential stroke using NIHSS or CPSS, the EMS documentation does not record whether the scales were used. As a result, we could not analyse the rate of CPSS or NIHSS use. Second, there were no data exchange between EMS and the hospital with the limitation of privacy rule and data safety. Hence, we could not analyse the accuracy of stroke identifications by EMS staff by comparing the initial prehospital screening with final patient diagnoses, leading to no evaluation of EMS hospital decision-making. Third, the databases did not include the data of eligible patients receiving thrombectomy, because emergency imaging assessment of intracranial vessels was not routinely used in all patients with stroke (only an NIHSS score of $\geq 7$ ). Further research on prehospital training, data and information security, as well as databases was still needed. In addition, our study period was only 2 years, and our results were unable to account for temporal trends in thrombolysis and thrombectomy rates. A further limitation is that this study only reviewed patients with stroke transported to 20 qualified hospitals, not all hospitals in Shenzhen with limited resources. Finally, our study was a retrospective single-centre study, and further multicentre prospective cohort studies are needed.

In summary, the article describes the key components of a city-wide stroke emergency map, such as certification of qualified local hospitals, identification of patients with stroke, acute stroke transport protocol and maintenance of the map, and highlights the successful implementation of a stroke emergency map in Shenzhen. For ischaemic stroke with suspected LVO, rapid identification of LVO and bypassing a nearby thrombolysis-capable centre directly to thrombectomy-capable hospitals indicated in the map may be reasonable, and further research should be encouraged. Telemedicine will play a key role in identifying appropriate patients needing more advanced treatment, optimising the referral system of prehospital emergency network. Additionally, future efforts should be conducted to improve public stroke awareness and EMS utilisation, which is a long-term repetition task.

Contributors LR, WL, SY and SH designed the study, analysed the data and prepared the main manuscript text and figures. SY, SH, ZHL, ZCL and YS collected the data. All authors have read and approved the final manuscript.

Funding This work was supported by the Science and Technology Innovation programme of Shenzhen Science and Technology Commission (grant number: JCYJ20151030151431727), Shenzhen Health and Population Family Planning Commission (grant number: SZLY2017012), and Shenzhen Development and Reform Commission's Stroke Screening and Prevention Public Service Platform improving programme.

Competing interests None declared.

Patient consent for publication Not required.
Ethics approval The study was approved by the Ethical Review Committee of the Shenzhen Second People's Hospital.

Provenance and peer review Not commissioned; externally peer reviewed. Data sharing statement No additional data are available.

Open access This is an open access article distributed in accordance with the Creative Commons Attribution Non Commercial (CC BY-NC 4.0) license, which permits others to distribute, remix, adapt, build upon this work non-commercially, and license their derivative works on different terms, provided the original work is properly cited, appropriate credit is given, any changes made indicated, and the use is non-commercial. See: http://creativecommons.org/licenses/by-nc/4.0/.

\section{REFERENCES}

1. Ng M, Fleming $\mathrm{T}$, Robinson $\mathrm{M}$, et al. Global, regional, and national prevalence of overweight and obesity in children and adults during 1980-2013: a systematic analysis for the Global Burden of Disease Study 2013. Lancet 2014;384:766-81.

2. GBD 2016 Mortality Collaborators. Global, regional, and national under-5 mortality, adult mortality, age-specific mortality, and life expectancy, 1970-2016: a systematic analysis for the Global Burden of Disease Study 2016. Lancet 2017;390:1084-150.

3. Liu L, Wang D, Wong KS, et al. Stroke and stroke care in China: huge burden, significant workload, and a national priority. Stroke 2011:42:3651-4.

4. Powers WJ, Rabinstein AA, Ackerson T, et al. Guidelines for the Early Management of Patients With Acute Ischemic Stroke: A Guideline for Healthcare Professionals From the American Heart Association/ American Stroke Association. Stroke 2018;2018:e46-e110.

5. Emberson J, Lees KR, Lyden P, et al. Effect of treatment delay, age, and stroke severity on the effects of intravenous thrombolysis with alteplase for acute ischaemic stroke: a meta-analysis of individual patient data from randomised trials. Lancet 2014;384:1929-35.

6. Wang $Y$, Liao $X$, Zhao $X$, et al. Using recombinant tissue plasminogen activator to treat acute ischemic stroke in China: analysis of the results from the Chinese National Stroke Registry (CNSR). Stroke 2011;42:1658-64.

7. Jin $\mathrm{H}$, Zhu S, Wei JW, et al. Factors associated with prehospital delays in the presentation of acute stroke in urban China. Stroke 2012;43:362-70.

8. Hachinski V, Donnan GA, Gorelick PB, et al. Stroke: working toward a prioritized world agenda. Int J Stroke 2010;5:238-56.

9. Kidwell CS, Starkman S, Eckstein M, et al. Identifying stroke in the field. Prospective validation of the Los Angeles prehospital stroke screen (LAPSS). Stroke 2000;31:71-6.

10. Ramanujam P, Guluma KZ, Castillo EM, et al. Accuracy of stroke recognition by emergency medical dispatchers and paramedics--San Diego experience. Prehosp Emerg Care 2008;12:307-13.

11. Riopelle RJ, Howse DC, Bolton C, et al. Regional access to acute ischemic stroke intervention. Stroke 2001;32:652-5.

12. Powers WJ, Derdeyn CP, Biller J, et al. American Heart Association/ American Stroke Association Focused Update of the 2013 Guidelines for the Early Management of Patients With Acute Ischemic Stroke Regarding Endovascular Treatment: A Guideline for Healthcare Professionals From the American Heart Association/American Stroke Association. Stroke 2015;2015:3020-35.

13. Higashida R, Alberts MJ, Alexander DN, et al. Interactions within stroke systems of care: a policy statement from the American Heart Association/American Stroke Association. Stroke 2013;44:2961-84.

14. Alberts MJ, Hademenos G, Latchaw RE, et al. Recommendations for the establishment of primary stroke centers. Brain Attack Coalition. JAMA 2000;283:3102-9.

15. Alberts MJ, Latchaw RE, Selman WR, et al. Recommendations for comprehensive stroke centers: a consensus statement from the Brain Attack Coalition. Stroke 2005;36:1597-616.

16. Albers GW, Marks MP, Kemp S, et al. Thrombectomy for Stroke at 6 to 16 Hours with Selection by Perfusion Imaging. 2018;378:708-18.

17. Nogueira RG, Jadhav AP, Haussen DC, et al. Thrombectomy 6 to 24 Hours after Stroke with a Mismatch between Deficit and Infarct. $N$ Engl J Med 2018;378:11-21.

18. Venema E, Boodt N, Berkhemer OA, et al. Workflow and factors associated with delay in the delivery of intra-arterial treatment for acute ischemic stroke in the MR CLEAN trial. J Neurointerv Surg 2018;10:424-8.

19. Ng FC, Low E, Andrew E, et al. Deconstruction of Interhospital Transfer Workflow in Large Vessel Occlusion: Real-World Data in the Thrombectomy Era. Stroke 2017;48:1976-9.

20. Saver JL, Goyal M, van der Lugt A, et al. Time to Treatment With Endovascular Thrombectomy and Outcomes From Ischemic Stroke: A Meta-analysis. JAMA 2016;316:1279-88. 
21. American Heart Association/American Stroke Association. SeverityBased Stroke Triage Algorithm for EMS. 2017 http://www.heart.org/ missionlifelinestroke (accessed 1 Dec 2017).

22. Barlinn J, Gerber J, Barlinn K, et al. Acute endovascular treatment delivery to ischemic stroke patients transferred within a telestroke network: a retrospective observational study. Int J Stroke 2017;12:502-9.
23. Kepplinger J, Dzialowski I, Barlinn K, et al. Emergency transfer of acute stroke patients within the East Saxony telemedicine stroke network: a descriptive analysis. Int J Stroke 2014;9:160-5.

24. Jiang $B, R u X$, Sun $H$, et al. Pre-hospital delay and its associated factors in first-ever stroke registered in communities from three cities in China. Sci Rep 2016;6:29795.

25. Zhao J, Liu R. Stroke 1-2-0: a rapid response programme for stroke in China. Lancet Neurol 2017;16:27-8. 